\title{
Electrochemical Study of
}

\section{a Heterobinuclear pyridylvinylidene Complex $\mathrm{Cp}(\mathrm{CO})_{2} \mathrm{Fe}\left[\mu-\mathrm{C}=\mathrm{CH}\left(2-\mathrm{C}_{5} \mathrm{H}_{4} \mathrm{~N}\right)\right] \mathrm{PdI}$}

\author{
Victor V. Verpekin*a, Galina V. Burmakina ${ }^{a, b}$, \\ Dmitry V. Zimonin ${ }^{a}$, Nina I. Pavlenko ${ }^{a}$, \\ Oleg. V. Semeikin' and Arkadii Z. Kreindlin ${ }^{\mathrm{c}}$ \\ anstitute of Chemistry and Chemical Technology SB RAS \\ FRC "Krasnoyarsk Science Center SB RAS” \\ 50/24 Akademgorodok, Krasnoyarsk, 660036, Russia \\ ${ }^{b}$ Siberian Federal University \\ 79 Svobodny, Krasnoyarsk, 660041, Russia \\ ${ }^{c} A . N$. Nesmeyanov Institute of Organoelement Compounds RAS \\ 28 Vavilova Str., Moscow, 119991, Russia
}

The redox properties of binuclear complex $\mathrm{Cp}(\mathrm{CO})_{2} \mathrm{Fe}\left[\mu-\mathrm{C}=\mathrm{CH}\left(2-\mathrm{C}_{5} \mathrm{H}_{4} \mathrm{~N}\right)\right] \mathrm{PdI}$ were studied by electrochemical methods at platinum, glassed carbon and dropping mercury electrodes in acetonitrile solution. It was found that a one-electron oxidation of the complex resulted in a cation-radical, which further undergoes decomposition, whereas a reduction gave an anion-radical, which further transforms into the 2-pirydylethynyl complex of iron $\mathrm{Cp}(\mathrm{CO})_{2} \mathrm{Fe}-\mathrm{C} \equiv \mathrm{C}-\left(2-\mathrm{C}_{5} \mathrm{H}_{4} \mathrm{~N}\right)$.

Keywords: electrochemistry, redox properties, binuclearcomplexes, $\mu$-pyridylvinylidene complexes, iron, palladium.

DOI: $10.17516 / 1998-2836-0038$.

(C) Siberian Federal University. All rights reserved

* Corresponding author E-mail address:vvv@sany-ok.ru; vvv@icct.ru 


\title{
Электрохимическое изучение
}

\section{гетеробиядерного пиридилвинилиденового комплекса $\mathrm{Cp}(\mathrm{CO})_{2} \mathrm{Fe}\left[\mu-\mathrm{C}=\mathrm{CH}\left(2-\mathrm{C}_{5} \mathrm{H}_{4} \mathrm{~N}\right)\right] \mathrm{PdI}$}

\author{
В.В. Верпекин ${ }^{\mathrm{a}}$, Г.В. Бурмакина ${ }^{\mathrm{a}, \tilde{0}}$, Д.В. Зимонин ${ }^{\mathrm{a}}$, \\ Н.И. Павленко ${ }^{\text {a }}$, О.В. Семейкин ${ }^{\mathrm{B}}$, А.З. Крейндлин ${ }^{\mathrm{B}}$ \\ ${ }^{a}$ Институт химии и химической технологии СО РАН \\ ФИЦ «Красноярский научный иентр СО РАН» \\ Россия, 660036, Красноярск, Академгородок, 50/24 \\ ${ }^{6}$ Сибирский федеральныий университет \\ Россия, 660041, Красноярск, пр. Свободный, 79 \\ ${ }^{6}$ Институт элементоорганической химии им. А.Н. Несмеянова \\ Россия, 119991, Москва, ул. Вавилова, 28
}

Электрохимическими методами изучены редокс-свойства биядерного комплекса $\mathrm{Cp}(\mathrm{CO})_{2} \mathrm{Fe}$ $\left[\mu-\mathrm{C}=\mathrm{CH}\left(2-\mathrm{C}_{5} \mathrm{H}_{4} \mathrm{~N}\right)\right] \mathrm{PdI}$ на платиновом, стеклоуглеродном и ртутном капельном электродах в аиетонитриле. Установлено, что одноэлектронное окисление комплекса приводит к образованию катион-радикала с последующим его разрушением, а восстановление - к анион-радикалу, превращающемуся в 2-пиридилэтинильный комплекс железа $\mathrm{Cp}(\mathrm{CO})_{2} \mathrm{Fe}-\mathrm{C} \equiv \mathrm{C}-\left(2-\mathrm{C}_{5} \mathrm{H}_{4} \mathrm{~N}\right)$.

Ключевые слова: электрохимия, редокс-свойства, биядерные комплексы, железо, палладий, н-пиридилвинилиденовые комплексы.

\section{Введение}

Одним из основных направлений развития современной химии является создание новых каталитических систем, изучение механизмов каталитических реакций и особенностей поведения катализаторов в них $[1,2]$. Среди многообразия катализаторов особое внимание уделяется соединениям палладия, катализирующим реакции кросс-сочетания углерод-углерод и углерод-гетероатом (кислород, сера, азот, фосфор и др.), и широко используемым в органическом синтезе $[3,4]$. В основе всех этих реакций лежат процессы окислительного присоединения и восстановительного элиминирования [2]. Кроме того, соединения палладия способны катализировать реакции сочетания переходный металл-углерод, что позволяет получать б-этинильные комплексы переходных металлов, представляющие научный и практический интерес как объекты для получения супрамолекулярных ансамблей в молекулярной электронике [5], нелинейной оптике [6, 7], а также как прекурсоры придилвинилиденовых комплексов [8] и потенциальные катализаторы электрохимического восстановления протона [9]. Среди известных реакций сочетания, катализируемых комплексами палладия, реакции сочетания металл-углерод являются наименее изученными [10]. В этих реакциях образуются полиядерные комплексы, которые в отдельных случаях удавалось зафиксировать и охарактеризовать [11]. В то же время гетеробиядерные комплексы с мостиковым $\mu$-пиридилвинилиденом извест- 
ны не были; среди близких по строению комплексов можно указать на полиядерные комплексы платины с мостиковым 2-пиридилэтинильным лигандом [12] и на полиядерные комплексы рутения с мостиковым $\mu_{4}-2$-пиридилвинилиденовым лигандом [13]. Электрохимическое поведение $\mu$-пиридилвинилиденовых комплексов ранее не было изучено.

Настоящая работа посвящена электрохимическому изучению редокс-свойств пиридилвинилиденового комплекса $\mathrm{Cp}(\mathrm{CO})_{2} \mathrm{Fe}\left[\mu-\mathrm{C}=\mathrm{CH}\left(2-\mathrm{C}_{5} \mathrm{H}_{4} \mathrm{~N}\right)\right] \mathrm{PdI}(\mathbf{1})$ в ацетонитриле на ртутном капельном (р.к.э), платиновом (Pt) и стеклоуглеродном (СУ) электродах методами полярографии, циклической вольтамперометрии (ЦВА) и электролиза при контролируемом потенциале.

Комплекс 1 получен взаимодействием циклопентадиенилдикарбонилиодида железа и 2-пиридилэтинила в смеси ТГФ/триэтиламин (2:1 по объему) в присутствии $\mathrm{PdCl}_{2}\left(\mathrm{PPh}_{3}\right)_{2}$ (10 мольных \%) и CuI (20 мольных \%) при комнатной температуре с выходом 3 \%. Методом рентгеноструктурного анализа установлена молекулярная структура комплекса 1 [14] (рис. 1).

Для установления схемы редокс-превращений биядерного комплекса 1 дополнительно в этих же экспериментальных условиях изучены редокс-свойства комплексов железа $\mathrm{Cp}(\mathrm{CO})_{2} \mathrm{FeI}$ (2), $\left[\mathrm{Cp}(\mathrm{CO})_{2} \mathrm{Fe}\right]_{2}(3), \mathrm{Cp}(\mathrm{CO})_{2} \mathrm{Fe}-\mathrm{C} \equiv \mathrm{C}-\left(2-\mathrm{C}_{5} \mathrm{H}_{4} \mathrm{~N}\right)(\mathbf{4})$ и $\mathrm{HC} \equiv \mathrm{C}-\left(2-\mathrm{C}_{5} \mathrm{H}_{4} \mathrm{~N}\right)(\mathbf{5})$. Электрохимия комплексов 2 и 3 подробно изучена [15, 16].

\section{Экспериментальная часть}

Комплекс $\mathrm{Cp}(\mathrm{CO})_{2} \mathrm{Fe}\left[\mu-\mathrm{C}=\mathrm{CH}\left(2-\mathrm{C}_{5} \mathrm{H}_{4} \mathrm{~N}\right)\right] \mathrm{PdI}$ (1) синтезировали по методике [14]. Электрохимические измерения проводили с использованием свежеприготовленных растворов соединений в ацетонитриле, очищенном по методике [17], в атмосфере аргона при комнатной температуpe. В качестве фонового электролита использовали $0.1 \mathrm{M} \mathrm{Et}_{4} \mathrm{NBF}_{4}$. Полярограммы и циклические вольтамперограммы (ЦВА) регистрировали на потенциостате IPC-Pro М (Вольта, СанктПетербург, Россия) с компьютерным и программным обеспечением по трехэлектродной схеме. В качестве рабочих электродов применяли ртутный капельный электрод с принудительным отрывом капель (m = 3.6 мг/c, $\tau=0.23$ с) и стационарные: платиновый электрод диаметром 1 мм

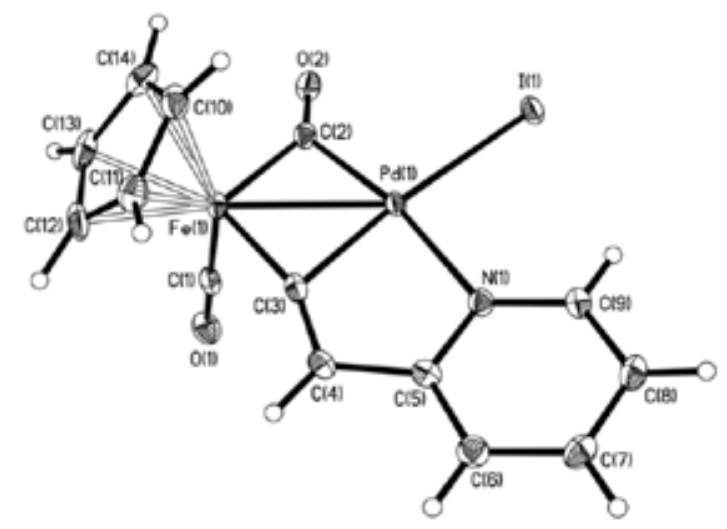

Рис. 1. Молекулярная структура комплекса $\mathrm{Cp}(\mathrm{CO})_{2} \mathrm{Fe}\left[\mu-\mathrm{C}=\mathrm{CH}\left(2-\mathrm{C}_{5} \mathrm{H}_{4} \mathrm{~N}\right)\right] \mathrm{PdI}$

Fig. 1. Molecular structure of $\mathrm{Cp}(\mathrm{CO})_{2} \mathrm{Fe}\left[\mu-\mathrm{C}=\mathrm{CH}\left(2-\mathrm{C}_{5} \mathrm{H}_{4} \mathrm{~N}\right)\right] \mathrm{PdI}$ 
или стеклоуглеродный электрод диаметром 5 мм, в тефлоновых корпусах диаметром 10 мм. ${ }^{1}$ Электродом сравнения служил полуэлемент $\mathrm{Ag} / 0.1 \mathrm{M} \mathrm{AgNO}_{3}$ в $\mathrm{MeCN}$, соединенный с ячейкой электролитическим мостом, заполненным фоновым электролитом, через капилляр Лунгина. В качестве вспомогательного электрода применяли платиновую спираль, помещенную в стеклянную трубку с пористым фильтром. Число электронов, участвующих в редокс-процессах, определяли путем сравнения высот волн исследуемых соединений с высотой одноэлектронной волны обратимого одноэлектронного окисления ферроцена.

Электролиз при контролируемом потенциале (ЭКП) проводили на потенциостате IPCPro М по трехэлектродной схеме. Рабочим электродом служила платиновая пластина, площадью $4 \mathrm{~cm}^{2}$. Электрод сравнения - $\mathrm{Ag} / 0.1 \mathrm{M} \mathrm{AgNO}_{3}$ в $\mathrm{MeCN}$, вспомогательный - платиновая спираль. Электролиз проводили при интенсивном барботировании аргоном. Регистрацию ИКспектров проводили на ИК-Фурье спектрометре Tensor 27 (Bruker).

\section{Результаты и обсуждение}

Электрохимические характеристики комплексов $\mathrm{Cp}(\mathrm{CO})_{2} \mathrm{Fe}\left[\mu-\mathrm{C}=\mathrm{CH}\left(2-\mathrm{C}_{5} \mathrm{H}_{4} \mathrm{~N}\right)\right] \mathrm{PdI}$ (1), $\mathrm{Cp}(\mathrm{CO})_{2} \mathrm{FeI}(\mathbf{2}),\left[\mathrm{Cp}(\mathrm{CO})_{2} \mathrm{Fe}\right]_{2}(3), \mathrm{Cp}(\mathrm{CO})_{2} \mathrm{Fe}-\mathrm{C} \equiv \mathrm{C}-\left(2-\mathrm{C}_{5} \mathrm{H}_{4} \mathrm{~N}\right)(\mathbf{4})$ и $\mathrm{HC} \equiv \mathrm{C}-\left(2-\mathrm{C}_{5} \mathrm{H}_{4} \mathrm{~N}\right)$ (5) в ацетонитриле на Pt-, CУ-, р.к.э. электродах приведены в таблице. Следует отметить, что значения редокс-потенциалов изученных комплексов и их устойчивость в редокс-реакциях зависят от материала электродов (табл.).

Комплекс 1 окисляется на Pt-, СУ- электродах и восстанавливается на Pt, СУ и р.к.э. в несколько стадий (табл.). На рис. 2(I) и 2(II) приведены его циклические вольтамперограммы в сравнении с таковыми для комплексов 2 и 3 на Pt и СУ электродах. Как видно из рис. 2(I) и 2(II), на циклических вольтамперограммах комплекса 1 наблюдаются три стадии восстановления и две окисления при скорости сканирования потенциала $25 \mathrm{mB} / \mathrm{c}$. Увеличение скорости сканирования потенциала до $200 \mathrm{MB} / \mathrm{c}$ приводит к исчезновению второй волны восстановления, что свидетельствует о том, что она принадлежит продукту распада одноэлектронного восстановления комплекса 1. Первая и третья волны восстановления при $\mathrm{E}_{1 / 2}=-1.52,-2.13$ В и первая волна окисления комплекса 1 на СУ-электроде, в отличие от окисления на Pt электроде, квазиобратимы (отношение предельных токов анодного и катодного пиков первой стадии восстановления $\mathrm{I}_{\mathrm{pa}} / \mathrm{I}_{\mathrm{pc}}=0.60$ (рис. 2(II), пики $\mathrm{A}_{1}^{\prime}, \mathrm{A}_{1}$ ), второй $\mathrm{I}_{\mathrm{pa}} / \mathrm{I}_{\mathrm{pc}}=0.90$ (рис. 2(II), пики $\mathrm{A}_{2}^{\prime}, \mathrm{A}_{2}$ ), катодного и анодного и пиков первой стадии окисления $\mathrm{I}_{\mathrm{pc}} / \mathrm{I}_{\mathrm{pa}}=0.77$ (рис. 2(II), пики $\mathrm{A}_{3}^{\prime}, \mathrm{A}_{3}$ ) при скорости сканирования потенциала $25 \mathrm{mB} / \mathrm{c})$.

Как видно из рис. 2 значение $\mathrm{E}_{1 / 2}$ одноэлектронного окисления комплекса $\mathrm{Cp}(\mathrm{CO})_{2} \mathrm{Fe}[\mu-$ $\left.\mathrm{C}=\mathrm{CH}\left(2-\mathrm{C}_{5} \mathrm{H}_{4} \mathrm{~N}\right)\right] \mathrm{PdI}(\mathbf{1})$ близко к потенциалу первой двухэлектронной волны окисления биядерного комплекса $\left[\mathrm{Cp}(\mathrm{CO})_{2} \mathrm{Fe}\right]_{2}(3)$ и протекает значительно легче, чем окисление $\mathrm{Cp}(\mathrm{CO})_{2} \mathrm{FeI}(2)$, в то время как значение $\mathrm{E}_{1 / 2}$ первой одноэлектронной волны восстановления комплекса 1 близко к потенциалу первой волны восстановления карбонилиодидного комплекса железа 2. Такое электрохимическое поведение гетерометаллического комплекса (1) может свидетельствовать о том, что при его окислении или восстановлении участвуют разные редокс-центры.

Использование различных рабочих электродов позволяет исследовать процессы окисления и восстановления соединений в более широкой области потенциалов. Так, рабочая область измерения потенциалов в ацетонитриле (относительно $\mathrm{Ag} / 0.1 \mathrm{M} \mathrm{AgNO}_{3}$ в $\mathrm{MeCN}$ ) на р.к.э. находится в пределах от 0.30 до -3.20 B, на Pt- и СУ-электродах от 2.0 до -2.2 В и от 2.0 до -2.6 В соответственно. 
Таблица. Электрохимические характеристики железо- и палладийсодержащих комплексов $(\mathrm{MeCN}$, $0.1 \mathrm{MEt}_{4} \mathrm{NBF}_{4}, 2 \mathrm{мM}, \mathrm{Ag} / 0.1 \mathrm{M} \mathrm{AgNO}_{3}$ в $\mathrm{MeCN}, \mathrm{C}=2 \mathrm{мM}, \mathrm{V}=25 \mathrm{mB} / \mathrm{c}$ )

Table. Electrochemical properties of iron- and palladium containing complexes $\left(\mathrm{MeCN}, 0.1 \mathrm{M} \mathrm{Et}_{4} \mathrm{NBF}_{4}, 2 \mathrm{MM}\right.$, $\mathrm{Ag} / 0.1 \mathrm{M} \mathrm{AgNO}_{3}$ in $\mathrm{MeCN}, \mathrm{C}=2 \mathrm{mM}, \mathrm{V}=25 \mathrm{mV} / \mathrm{s}$ )

\begin{tabular}{|c|c|c|c|c|c|}
\hline \multirow{3}{*}{ Соединение } & \multicolumn{5}{|c|}{$\mathrm{E}_{1 / 2}, \mathrm{~B}(\mathrm{n})$} \\
\hline & \multicolumn{2}{|c|}{$\mathrm{Pt}$} & \multicolumn{2}{|c|}{ Cy } & \multirow{2}{*}{$\begin{array}{c}\text { Р.к.э. } \\
\text { Red }\end{array}$} \\
\hline & $\mathrm{Ox}$ & Red & $\mathrm{Ox}$ & Red & \\
\hline $\mathrm{Cp}(\mathrm{CO})_{2} \mathrm{Fe}\left[\mu-\mathrm{C}=\mathrm{CH}\left(2-\mathrm{C}_{5} \mathrm{H}_{4} \mathrm{~N}\right)\right] \mathrm{PdI}(\mathbf{1})$ & $\begin{array}{l}0.22(1) \\
1.27(2)\end{array}$ & $\begin{array}{c}-1.42(1) \\
-1.68(<<1) \\
-1.97(1)\end{array}$ & $\begin{array}{l}0.31^{\mathrm{a}}(1) \\
1.37(<1)\end{array}$ & $\begin{array}{c}-1.52^{\mathrm{a}}(1) \\
-1.81(<<1) \\
-2.13^{\mathrm{a}}(1)\end{array}$ & $\begin{array}{l}-1.44(2) \\
-2.24(1) \\
-2.60(<1) \\
-2.90(<1)\end{array}$ \\
\hline $\mathrm{Cp}(\mathrm{CO})_{2} \mathrm{FeI}(\mathbf{2})$ & $\begin{array}{c}0.80(<2) \\
1.39(1)\end{array}$ & $-1.33(2)$ & $0.94^{\mathrm{a}}(2)$ & $\begin{array}{l}-1.36^{\mathrm{a}}(1) \\
-2.18^{\mathrm{a}}(1)\end{array}$ & $\begin{array}{l}-1.42(2) \\
-2.47(1)\end{array}$ \\
\hline$\left[\mathrm{Cp}(\mathrm{CO})_{2} \mathrm{Fe}\right]_{2}(\mathbf{3})$ & $0.36(2)$ & - & $0.23(2)$ & $-2.12^{\mathrm{a}}(2)$ & $\begin{array}{l}-2.20(2) \\
-2.60(1)\end{array}$ \\
\hline $\mathrm{Cp}(\mathrm{CO})_{2} \mathrm{Fe}-\mathrm{C} \equiv \mathrm{C}-\left(2-\mathrm{C}_{5} \mathrm{H}_{4} \mathrm{~N}\right)(4)$ & $0.81(1)$ & $-2.10(1)$ & $\begin{array}{l}0.87(1) \\
1.49(1)\end{array}$ & $-2.16^{\mathrm{a}}(1)$ & $\begin{array}{l}-2.25(1) \\
-2.60(1) \\
-2.95(<1)\end{array}$ \\
\hline $\mathrm{HC} \equiv \mathrm{C}-\left(2-\mathrm{C}_{5} \mathrm{H}_{4} \mathrm{~N}\right)$ & - & - & - & - & $\begin{array}{l}-2.80(1) \\
-2.93(1)\end{array}$ \\
\hline
\end{tabular}

Примечание: ${ }^{\mathrm{a}}$ - квазиобратимая стадия; $\mathrm{n}$ - число электронов, участвующих в электрохимической стадии (знак «<» означает, что высота волны исследуемого соединения меньше, чем высота одноэлектронной волны).

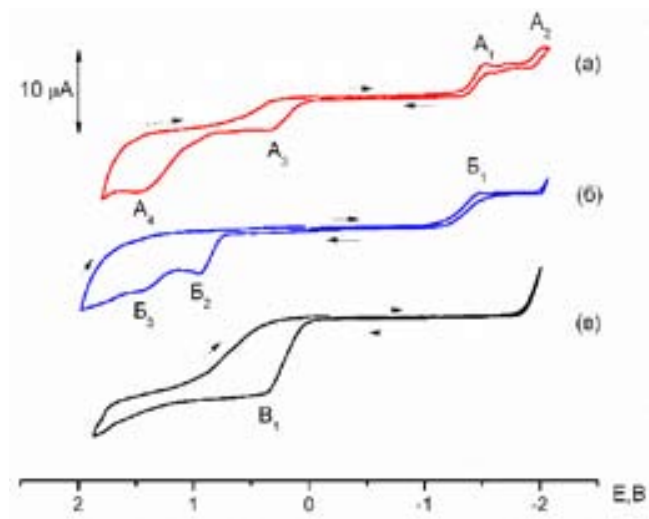

(I)

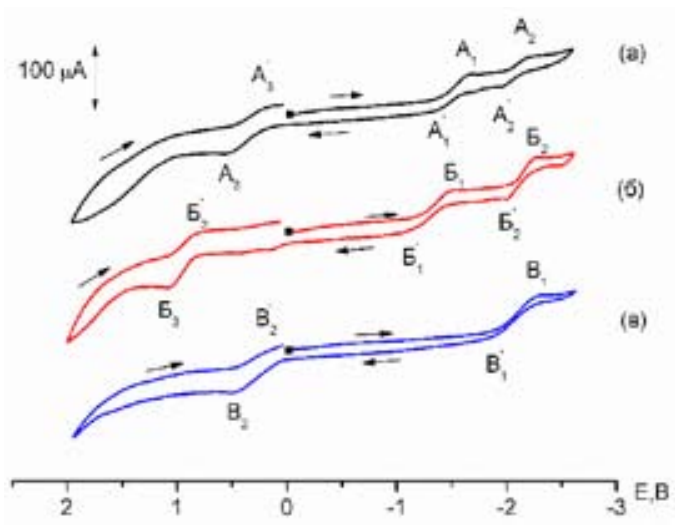

(II)

Рис. 2. Циклические вольтамперограммы комплексов: a - $\mathrm{Cp}(\mathrm{CO})_{2} \mathrm{Fe}\left[\mu-\mathrm{C}=\mathrm{CH}\left(2-\mathrm{C}_{5} \mathrm{H}_{4} \mathrm{~N}\right)\right] \mathrm{PdI}$ (1), б - $\mathrm{Cp}(\mathrm{CO})_{2} \mathrm{FeI}(\mathbf{2}), \mathbf{в}-\left[\mathrm{Cp}(\mathrm{CO})_{2} \mathrm{Fe}\right]_{2}(\mathbf{3}),(\mathrm{I})$ - на Pt, (II) - на СУ-электродах $\left(\mathrm{MeCN}, 0.1 \mathrm{MEt}_{4} \mathrm{NBF}_{4}, \mathrm{Ag} / 0.1 \mathrm{M}\right.$ $\mathrm{AgNO}_{3}$ в $\left.\mathrm{MeCN}, \mathrm{C}=2 \mathrm{mM}, \mathrm{V}=25 \mathrm{mB} / \mathrm{c}\right)$

Fig. 2. Cyclic voltammogramms of a $-\mathrm{Cp}(\mathrm{CO})_{2} \mathrm{Fe}\left[\mu-\mathrm{C}=\mathrm{CH}\left(2-\mathrm{C}_{5} \mathrm{H}_{4} \mathrm{~N}\right)\right] \mathrm{PdI}$ (1), б - $\mathrm{Cp}(\mathrm{CO})_{2} \mathrm{FeI}$ (2), B- $\left[\mathrm{Cp}(\mathrm{CO})_{2} \mathrm{Fe}\right]_{2}(\mathbf{3}) ;(\mathrm{I})$-at Pt, (II) - at GC electrodes $\left(\mathrm{MeCN}, 0.1 \mathrm{MEt}_{4} \mathrm{NBF}_{4}, \mathrm{Ag} / 0.1 \mathrm{M} \mathrm{AgNO}_{3}\right.$ in MeCN, C=2 mM, $\mathrm{V}=25 \mathrm{mV} / \mathrm{s})$

Известно $[15,16]$, что необратимое двухэлектронное окисление комплекса 3 протекает с разрывом связи $\mathrm{Fe}-\mathrm{Fe}$ и приводит к образованию катионов $\left[\mathrm{Cp}(\mathrm{CO})_{2} \mathrm{Fe}(\mathrm{NCMe})\right]^{+}$по схеме

$$
\begin{aligned}
{\left[\mathrm{Cp}(\mathrm{CO})_{2} \mathrm{Fe}\right]_{2}+2 \mathrm{MeCN} } & \stackrel{-2 \mathrm{e}^{-}}{\longrightarrow} 2\left[\mathrm{Cp}(\mathrm{CO})_{2} \mathrm{Fe}(\mathrm{NCMe})\right]^{+} . \\
& -432-
\end{aligned}
$$


Образующийся в результате необратимого окисления комплекса 3 при $\mathrm{E}_{\mathrm{p}}{ }^{\mathrm{a}}=0.55 \mathrm{~B}$ (СУ, $\mathrm{MeCN}, 0,1 \mathrm{M}$ TBAH, отн. $\mathrm{Ag} / \mathrm{AgCl})$ катион $\left[\mathrm{Cp}(\mathrm{CO})_{2} \mathrm{Fe}(\mathrm{NCMe})\right]^{+}$, при обратном сканировании потенциала при скорости $250 \mathrm{MB} / \mathrm{c}$, дает волну восстановления при $\mathrm{E}_{\mathrm{p}}{ }^{\mathrm{c}}=-1.01 \mathrm{~B}$ [15]. В наших условиях двухэлектронное окисление комплекса 3 на СУ-электроде ( $\mathrm{V}=200 \mathrm{mB} / \mathrm{c})$ наблюдается при $\mathrm{E}_{1 / 2}=0,23 \mathrm{~B}$, восстановление $\left[\mathrm{Cp}(\mathrm{CO})_{2} \mathrm{Fe}(\mathrm{NCMe})\right]^{+}-$при $\mathrm{E}_{1 / 2}=-1,06 \mathrm{~B}$.

При обратном сканировании потенциала при высоких скоростях на ЦВА гетерометаллического комплекса 1 не удалось зафиксировать волну восстановления катиона $\left[\mathrm{Cp}(\mathrm{CO})_{2} \mathrm{Fe}(\mathrm{NCMe})\right]^{+}$. При этом в ИК-спектрах продуктов электролиза при контролируемом потенциале предельного тока первой волны окисления комплекса 1 не обнаружено полос поглощения валентных колебаний карбонильных групп как исходного FePd комплекса, так и возможных продуктов его превращений, что свидетельствует об его полном разложении после одноэлектронного окисления. На основании полученных данных можно предположить, что квазиобратимое одноэлектронное окисление комплекса 1 приводит к образованию относительно устойчивого катион-радикала $\mathbf{1}^{+*}$, который в дальнейшем распадается с образованием соединений железа и палладия и ряда органических соединений.

Значения $\mathrm{E}_{1 / 2}$ первой волны восстановления комплекса 1 практически совпадают на р.к.э. и $\mathrm{Pt}$-электродах и близки к потенциалу восстановления комплекса $\mathrm{Cp}(\mathrm{CO})_{2} \mathrm{FeI}$ (2) на р.к.э. (табл.), что свидетельствует о том, что до начала восстановления комплекса 1 не происходит его взаимодействия с материалом электрода. Более того, контакт раствора комплекса $\mathbf{1}$ со ртутью не приводит к изменению его ИК-спектров, что свидетельствует о том, что отрыв йодид-иона происходит после его одноэлектронного восстановления на р.к.э.

Известно, что металлоорганические комплексы переходных металлов, содержащие б-связь металл-галоген, восстанавливаются с разрывом связи металл-галоген и образованием металлоорганических радикалов, устойчивость которых зависит от природы металла и лиганда [16]. Так, железокарбонильный комплекс 2 восстанавливается по следующей схеме:

$$
\mathrm{Cp}(\mathrm{CO})_{2} \mathrm{FeI} \stackrel{+\overline{\mathrm{e}}}{\rightarrow}\left[\mathrm{Cp}(\mathrm{CO})_{2} \mathrm{Fe}\right]^{\cdot}+\mathrm{I}^{-}
$$

Восстановление палладийсодержащего комплекса $\mathrm{C}_{3} \mathrm{H}_{5} \mathrm{PdI}$ протекает по схеме [18]:

$$
\mathrm{C}_{3} \mathrm{H}_{5} \mathrm{PdI} \stackrel{+\overline{\mathrm{e}}}{\rightarrow}\left[\mathrm{C}_{3} \mathrm{H}_{5} \mathrm{Pd}\right]^{\cdot}+\mathrm{I}^{-} .
$$

Восстановление комплекса $\mathrm{PdCl}_{2}\left(\mathrm{PPh}_{3}\right)_{2}$ идет с присоединением двух электронов и элиминированием хлорид-иона [19]:

$$
\begin{aligned}
& \mathrm{PdCl}_{2}\left(\mathrm{PPh}_{3}\right)_{2} \stackrel{+2 \overline{\mathrm{e}}}{\longrightarrow}\left[\mathrm{PdCl}\left(\mathrm{PPh}_{3}\right)_{2}\right]^{-}+\mathrm{Cl}^{-} \\
& {\left[\mathrm{PdCl}\left(\mathrm{PPh}_{3}\right)_{2}\right]^{-} \rightarrow\left[\mathrm{Pd}_{2}(\mu-C l)_{2}\left(\mathrm{PPh}_{3}\right)_{4}\right]^{2-}}
\end{aligned}
$$

Би- и трехъядерные карбонилгалогениды осмия и рения, содержащие терминальные атомы галогенов, также восстанавливаются с отрывом галогенид-ионов на первой одно- или двухэлектронной стадии [20-22].

Таким образом, можно предположить, что квазиобратимое одноэлектронное восстановление комплекса 1 приводит к образованию относительно устойчивого анион-радикала 1·-

$$
-433-
$$




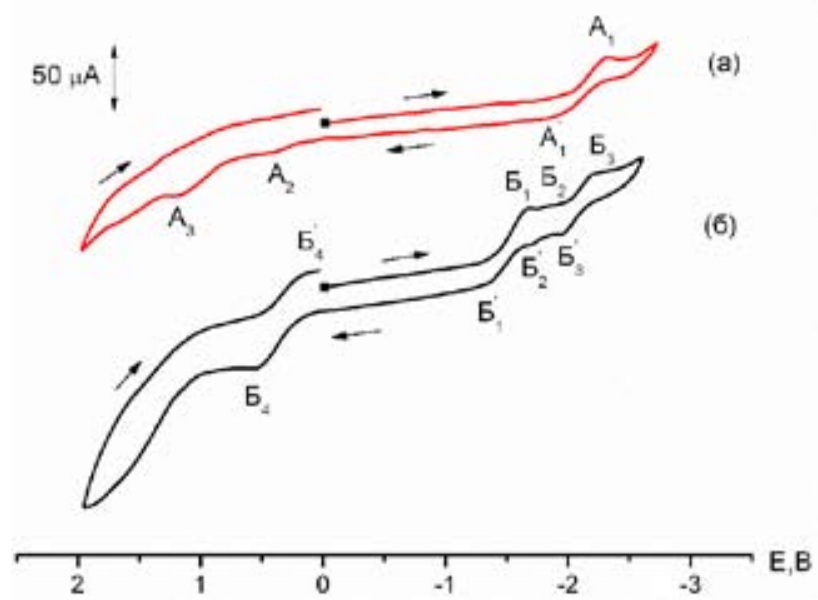

Рис. 3. Циклические вольтамперограммы комплексов: a $-\mathrm{Cp}(\mathrm{CO})_{2} \mathrm{Fe}-\mathrm{C} \equiv \mathrm{C}-\left(2-\mathrm{C}_{5} \mathrm{H}_{4} \mathrm{~N}\right)(\mathbf{4})$; $\boldsymbol{\boldsymbol { \sigma }}-\mathrm{Cp}(\mathrm{CO})_{2} \mathrm{Fe}$ $\left[\mu-\mathrm{C}=\mathrm{CH}\left(2-\mathrm{C}_{5} \mathrm{H}_{4} \mathrm{~N}\right)\right] \mathrm{PdI}(\mathbf{1}) ;\left(\mathrm{CV}, \mathrm{MeCN}, 0.1 \mathrm{MEt}_{4} \mathrm{NBF}_{4}, \mathrm{Ag} / 0.1 \mathrm{M} \mathrm{AgNO}_{3}\right.$ в $\left.\mathrm{MeCN}_{2} \mathrm{C}=2 \mathrm{мM}, \mathrm{V}=25 \mathrm{мB} / \mathrm{c}\right)$

Fig. 3 Cyclic voltammogramms of $\mathbf{a}-\mathrm{Cp}(\mathrm{CO})_{2} \mathrm{Fe}-\mathrm{C} \equiv \mathrm{C}-\left(2-\mathrm{C}_{5} \mathrm{H}_{4} \mathrm{~N}\right)(\mathbf{4}), \boldsymbol{\sigma}-\mathrm{Cp}(\mathrm{CO}){ }_{2} \mathrm{Fe}\left[\mu-\mathrm{C}=\mathrm{CH}\left(2-\mathrm{C}_{5} \mathrm{H}_{4} \mathrm{~N}\right)\right] \mathrm{PdI}(\mathbf{1})$ (GC, $\mathrm{MeCN}, 0.1 \mathrm{M} \mathrm{Et}_{4} \mathrm{NBF}_{4}, \mathrm{Ag} / 0.1 \mathrm{M} \mathrm{AgNO}_{3}$ in $\mathrm{MeCN}, \mathrm{C}=2 \mathrm{mM}, \mathrm{V}=25 \mathrm{mV} / \mathrm{s}$ )

дальнейшее превращение которого приводит к элиминированию йодид-иона и образованию радикала (1-I):

На рис. 3 приведены ЦВА комплексов $\mathrm{Cp}(\mathrm{CO})_{2} \mathrm{Fe}\left[\mu-\mathrm{C}=\mathrm{CH}\left(2-\mathrm{C}_{5} \mathrm{H}_{4} \mathrm{~N}\right)\right] \mathrm{PdI}(\mathbf{1})$ и $\mathrm{Cp}(\mathrm{CO})_{2} \mathrm{Fe}-$ $\mathrm{C} \equiv \mathrm{C}-\left(2-\mathrm{C}_{5} \mathrm{H}_{4} \mathrm{~N}\right)$. Как видно из рис. 3 значение потенциала третьей волны восстановления комплекса 1 близко к потенциалу первой волны восстановления 2-пиридилэтинильного комплекса железа 4.

Следовательно, образующийся на первой стадии восстановления комплекса 1 радикал (1-I) распадается с образованием металлического палладия и $19 \overline{\mathrm{e}}$ пиридилвинилиденового комплекса железа, который быстро превращается во время съемки ЦВА в стабильный $18 \overline{\mathrm{e}}$ пиридилалкинильный комплекс железа с отщеплением водорода. На основании полученных результатов предложена следующая схема восстановления комплекса $\mathbf{1}$ :

$\mathrm{Cp}(\mathrm{CO})_{2} \mathrm{Fe}\left[\mu-\mathrm{C}=\mathrm{CH}\left(2-\mathrm{C}_{5} \mathrm{H}_{4} \mathrm{~N}\right)\right] \mathrm{PdI} \underset{-\overline{\mathrm{e}}}{\stackrel{+\overline{\mathrm{e}}}{\rightarrow}}\left\{\mathrm{Cp}(\mathrm{CO})_{2} \mathrm{Fe}\left[\mu-\mathrm{C}=\mathrm{CH}\left(2-\mathrm{C}_{5} \mathrm{H}_{4} \mathrm{~N}\right)\right] \mathrm{PdI}\right\}^{\cdot-}$

$\left\{\mathrm{Cp}(\mathrm{CO})_{2} \mathrm{Fe}\left[\mu-\mathrm{C}=\mathrm{CH}\left(2-\mathrm{C}_{5} \mathrm{H}_{4} \mathrm{~N}\right)\right] \mathrm{PdI}\right\}^{\cdot-} \rightarrow\left[\mathrm{Cp}(\mathrm{CO})_{2} \mathrm{Fe}\left[\mu-\mathrm{C}=\mathrm{CH}\left(2-\mathrm{C}_{5} \mathrm{H}_{4} \mathrm{~N}\right)\right] \mathrm{PdI}\right]^{\cdot}+\mathrm{I}^{-}$

$\left[\mathrm{Cp}(\mathrm{CO})_{2} \mathrm{Fe}\left[\mu-\mathrm{C}=\mathrm{CH}\left(2-\mathrm{C}_{5} \mathrm{H}_{4} \mathrm{~N}\right)\right] \mathrm{PdI}\right]^{\cdot} \rightarrow\left[\mathrm{Cp}(\mathrm{CO})_{2} \mathrm{Fe}=\mathrm{C}=\mathrm{CH}\left(2-\mathrm{C}_{5} \mathrm{H}_{4} \mathrm{~N}\right)\right]^{\cdot}+\mathrm{Pd}^{0}$

$\left[\mathrm{Cp}(\mathrm{CO})_{2} \mathrm{Fe}=\mathrm{C}=\mathrm{CH}\left(2-\mathrm{C}_{5} \mathrm{H}_{4} \mathrm{~N}\right)\right]^{\cdot} \rightarrow \mathrm{Cp}(\mathrm{CO})_{2} \mathrm{Fe}-\mathrm{C} \equiv \mathrm{C}\left(2-\mathrm{C}_{5} \mathrm{H}_{4} \mathrm{~N}\right)+\mathrm{H}^{\cdot}$

Однако электролиз при контролируемом потенциале предельного тока первой волны восстановления комплекса 1 провести не удалось из-за пассивации поверхности электрода образующимся в ходе электрохимической реакции металлическим палладием.

По результатам электрохимического изучения гетерометаллического пиридилвинилиденового комплекса $\mathrm{Cp}(\mathrm{CO})_{2} \mathrm{Fe}\left[\mu-\mathrm{C}=\mathrm{CH}\left(2-\mathrm{C}_{5} \mathrm{H}_{4} \mathrm{~N}\right)\right] \mathrm{PdI}$ (1) установлено, что одноэлектронное окисление и одноэлектронное восстановление комплекса 1 приводит к образованию 
относительно устойчивых катион- и анион-радикалов. Катион-радикал $\mathbf{1}^{+}$распадается с образованием соединений железа и палладия, образующих нерастворимый осадок, и ряда неидентифицированных органических соединений. Дальнейшие превращения анион-радикала 1- приводят к образованию металлического палладия и пиридилалкинильного комплекса железа. Таким образом, редуктивная активация комплекса приводит к декоординации палладиевого фрагмента и образованию пиридилалкинильного комплекса $\mathrm{Cp}(\mathrm{CO})_{2} \mathrm{Fe}-\mathrm{C} \equiv$ $\equiv \mathrm{C}-\left(2-\mathrm{C}_{5} \mathrm{H}_{4} \mathrm{~N}\right)$.

Исследование выполнено при финансовой поддержке Российского фонда фундаментальных исследований, Правительства Красноярского края, Красноярского краевого фонда поддержки научной и научно-технической деятельности в рамках научного проекта № 16-43-243078.

\section{Список литературы}

1. Hartwig J.F. Organotransition Metal Chemistry. From Bonding to Catalysis. first. Casebound: University Science Books, 2010. 1160 p.

2. Meijere A. de, Diederich F. Metal-Catalyzed Cross-Coupling Reactions. 2d ed.; ed. Meijere A. de, Diederich F. WILEY-VCH Verlag GmbH \& Co. KGaA, 2004. Vol. 1. 916 p.

3. Chinchilla R., Najera C. The Sonogashira reaction: A booming methodology in synthetic organic chemistry. Chem. Rev. 2007. Vol. 107(3), P. 874-922.

4. Chinchilla R., Najera C. Recent advances in Sonogashira reactions. Chem. Soc. Rev. 2011. Vol. 40(10), P. 5084-5121.

5. Le Stang S. et al. New pyridyl-functionalized organoiron alkynyl complexes. Easy access to polymetallic architectures featuring an electroactive site by simple co-ordination reactions. J. Organomet. Chem. 1999. Vol. 572(2), P. 189-192.

6. Medei L. et al. A joint experimental and computational study on the electronic communication in diethynylaryl-bridged $\left(\eta^{5}-\mathrm{C}_{5} \mathrm{H}_{5}\right) \mathrm{Fe}\left(\eta^{2}\right.$-dppe) and $\left(\eta^{5}-\mathrm{C}_{5} \mathrm{H}_{5}\right) \mathrm{Fe}(\mathrm{CO})_{2}$ units. Eur. J. Inorg. Chem. 2006. № 13, P. 2582-2597.

7. Long N.J., Williams C.K. Metal alkynyl $\sigma$ complexes: Synthesis and materials. Angew. Chemie - Int. Ed. 2003. Vol. 42(23), P. 2586-2617.

8. Chou H.H. et al. Reactions of ruthenium acetylide and vinylidene complexes containing a 2-pyridyl group.Organometallics 2008. Vol. 27(20), P. 5212-5220.

9. Valyaev D.A. et al. Proton reduction catalysis by manganese vinylidene and allenylidene complexes. J. Organomet. Chem. 2007. Vol. 692(15), P. 3207-3211.

10. Crescenzi R., Sterzo C.L. Synthesis of Metal Acetylides via Palladium-Catalyzed CarbonMetal Bond Formation. Organometallics 1992. Vol. 11, P. 4301-4305.

11. Ricci A., Lo Sterzo C. A new frontier in the metal-catalyzed cross-coupling reaction field. The palladium-promoted metal-carbon bond formation. Scope and mechanism of a new tool in organometallic synthesis. J. Organomet. Chem. 2002. Vol. 653(1-2), P. 177-194.

12. Berenguer R. et al. Reactivity of $\left[\operatorname{trans}-\mathrm{PtH}\left(\mathrm{C} \equiv \mathrm{CC}_{5} \mathrm{H}_{4} \mathrm{~N}-2\right)\left(\mathrm{PPh}_{3}\right)_{2}\right]$ toward [cis- $\left.\mathrm{Pt}\left(\mathrm{C}_{6} \mathrm{~F}_{5}\right)_{2}(\mathrm{thf})_{2}\right]$. Synthesis of an unusual triplatinum cluster-substituted Platinum complex. Organometallics. 1999. Vol. 18, P. 1653-1662. 
13. Azam K.A. et al. Double Carbon-Hydrogen Activation of 2-Vinylpyridine: Synthesis of tri- and pentanuclear clusters containing the $\mu-\mathrm{NC}_{5} \mathrm{H}_{4} \mathrm{CH}=\mathrm{C}$ ligand. Organometallics. 2008. Vol. 27, P. 5163-5166.

14. Verpekin V.V. et al. Crystal structure of $\mu$-carbonyl- $1: 2 \kappa^{2} \mathrm{C}: \mathrm{C}$-carbonyl- $1 \kappa \mathrm{C}-\left(1 \eta^{5}-\right.$ cyclopentadienyl)iodido-2 $\kappa \mathrm{I}-\quad\left[\mu-2\right.$-(pyridin-2-yl)ethene-1,1-diyl- $\left.1 \kappa \mathrm{C}^{1}: 2 \kappa^{2} \mathrm{~N}, \mathrm{C}^{1}\right]$ ironpalladium( $\mathrm{Fe}-$ Pd) benzene monosolvate. Acta Crystallogr. Sect. E Crystallogr. Commun. 2017. Vol. 73(1), P. $68-71$.

15. Bullock J.P., Palazotto M.C., Mann K.R. Electrochemistry and infrared spectroelectrochemistry of $\left[\left(\eta^{5}-\mathrm{C}_{5} \mathrm{R}_{5}\right) \mathrm{Fe}(\mathrm{CO})_{2}\right]_{2}(\mathrm{R}=\mathrm{H}, \mathrm{Me})$ : generation and characterization of $\left[\left(\eta^{5}-\mathrm{C}_{5} \mathrm{R}_{5}\right) \mathrm{Fe}(\mathrm{CO})_{2}\right]_{2}\left(\mathrm{PF}_{6}\right)$ complexes. Inorg. Chem. 1991. Vol. 30(6), P. 1284-1293.

16. Губин С.П., Денисович Л.И. Электрохимическое исследование окислительновосстановительных реакций $\pi$-комплексов и металлоорганических соединений переходных металлов. Итоги науки и техники. Электрохимия. М.: ВИНИТИ, 1978. Т. 13. С. 94-154. [Gubin S.P., Denisovich L.I. Electrochemical study of oxidation and reduction reactions of $\pi$-complexes and organometallic compounds of transition metals. Results of science and technique. Electrochemistry. Moscow. VINITI. 1978. Vol. 13, P. 94-154. (In Russ.)]

17. O'Donnell J.T., Ayres J.T., Mann C.K. Preparation of high purity acetonitrile. Anal. Chem. 1965. Vol. 37(9), P. 1161-1162.

18. Губин С.П., Денисович Л.И. $\gamma$-Замещенные бисацетилактонаты палладия. Полярография, спектры ИК и УФ. Изв. АН СССР. Сер. хим. 1966. № 1, С. 149-151. [Gubin S.P., Denisovich L.I. $\gamma$-Substituted bisacetylactonates of palladium. Polarography, IR and UV spectra. Izv. Akad. Nauk SSSR, Ser. Khim. 1966. N. 1, P. 149-151. (In Russ.)]

19. Amatore C., Jutand A. Anionic Pd (0) and Pd (II) intermediates in Palladium-catalyzed Heck and Cross-coupling reactions. Acc. Chem. Res. 2000. Vol. 33(5), P. 314-321.

20. Губин С.П., Трухачева В.А., Бурмакина Г.В., Ченцова О.М., Иогансон А.А. Превращение металлоцепь - металлоцикл при восстановлении трехъядерных карбонилгалогенидовосмия. Изв. АНСССР. Сер. хим. 1981. № 11, С. 2423-2428. [Gubin S.P., Trukhacheva V.A., Burmakina G.V., Chernsova O.M., Ioganson A.A. Metallochain - metallocycle transformation of trinuclear carbonylhalogenides of osmium under reduction. Izv. Akad. Nauk SSSR, Ser. Khim. 1981. N. 11, P. 2423-2428 (In Russ.)]

21. Бурмакина Г.В., Трухачева В.А., Губин С.П. О ковалентном характере связей металлгалоген в трехъядерных карбонилгалогенидах осмия. Изв. АНСССР. Сер. хим. 1990. № 2, С. 459460. [Burmakina G.V., Trukhacheva V.A., Gubin S.P. About covalent nature of metal-halogen bonds in trinuclear carbonylhalogenides of osmium. Izv. Akad. Nauk SSSR, Ser. Khim. 1990. No. 2, P. 459-460 (In Russ.)]

22 Губин С.П., Бурмакина Г.В., Максаков В.А., Павленко Н.И., Баулина О.В., Кирин В.П. Кластерные металлгалогениды. Электрохимическое восстановление карбонилиодидов осмия. Коорд. химия 1995.T. 21(2), C. 140-143. [Gubin S.P., Burmakina G.V., Maksakov V.A., Pavlenko N.I., Baulina O.V., Kirin V.P. Electrochemical reduction of carbonylhalogenides of osmium. Russian Journal of Coordination chemistry 1995. Vol. 21(2), P. 140-143 (In Russ.)] 